\title{
Evaluation of respiratory model employing conventional NIH mice to access the immunity induced by cellular and acellular pertussis vaccines
}

\author{
Alexandre Alves de Souza de Oliveira Dias $/{ }^{+}$, Maria Aparecida Affonso Boller, \\ Lúcia Maria Correa Werneck, Raphael Hirata Junior*, Ana Luíza Mattos-Guaraldi*
}

Laboratório de Vacinas Bacterianas, Departamento de Imunologia, Instituto Nacional de Controle de Qualidade em Saúde-Fiocruz, Av. Brasil 4365, 21040-900 Rio de Janeiro, RJ, Brasil *Faculdade de Ciências Médicas, Universidade do Estado do Rio de Janeiro, Rio de Janeiro, RJ, Brasil

The increasing number of pertussis cases reported on the last twenty years and the existence of new acellular vaccines reinforce the need of research for experimental models to assure the quality of available pertussis vaccines. In this study, allotments of whole-cell and acellular pertussis vaccines were tested through the Intranasal Challenge Model (INM) using conventional NIH mice. The results have been compared to those achieved by the "Gold standard" Intracerebral Challenge Model (ICM). In contrast to ICM, INM results did not show intralaboratorial variations. Statistical analysis by Anova and Ancova tests revealed that the INM presented reproducibility and allowed identification and separation of different products, including three-component and four-component accellular pertussis vaccines. INM revealed differences between pertussis vaccines. INM provides lower distress to the mice allowing the reduction of mice number including the possibility of using conventional mice (less expensive) under non-aseptic environment. Thus, INM may be used as an alternative method of verifying the consistence of allotment production, including acellular pertussis vaccines.

Key words: pertussis - vaccine - intranasal challenge model - NIH conventional mice

Pertussis continues to be a significant cause of morbidity and mortality among nonimmunized young infants worldwide. Reported cases of pertussis represent only a fraction of the actual number of infections. Possible explanations for failure to diagnose pertussis include the heterogeneity in pertussis disease expression and low physician awareness and index suspicion, including poorly performed laboratorial tests or their lack of availability (Guiso 2001, Cherry et al. 2005).

Due to the side effects of whole-cell pertussis vaccines $(\mathrm{Pw})$ and the difficulties to produce them in a reproducible manner, researches were undertaken in order to develop new pertussis vaccines as effective but better tolerated. Although the implementation of childhood pertussis immunization programs has significantly reduced the occurrence of the disease in children, there is a change on transmission instead child to child transmission. Waning vaccine-induced immunity permits the disease to affect adolescents and adults, who in turn transmit pertussis to unimmunized and incompletely immunized infants, showing changes in disease epidemiology. For this reason $\mathrm{Pw}$ were quickly replaced by acellular pertussis vaccines $(\mathrm{Pa})$ and boosters were introduced for adolescents and adults (Forsyth et al. 2005, Greenberg \& Caro 2005, Tan \& Plotkin 2005). Due to this change in pertussis vac-

Financial support: INCQS-Fiocruz/MS

${ }^{+}$Corresponding author: alexandre.dias@incqs.fiocruz.br

Received 25 April 2006

Accepted 13 September 2006 cine it is now important to evaluate the reproducibility of the new vaccines.

Besides the ethical question on doing clinical studies employing nonimmunized infants with new formulas proposed every year, the increasing number of reported pertussis cases reinforces the need to attempt new methodologies to assure the immunogenic activity for vaccines against Bordetella pertussis (Eude et al. 1999, Guiso et al. 1999, Canthaboo et al. 2000a,b). These facts emphasize the need of searching for experimental models to efficiently demonstrate relation on field, bringing information about the mechanisms and agents involved in human disease (Mills et al. 1998).

The recently proposed Intranasal Model (INM) is a test relatively simple, with accuracy, and with high reproducibility. In contrast to Intracerebral Model (ICM), this respiratory model presents the possibility to evaluate acellular vaccines available in the market as news formulations in terms of the antigen composition and their concentration (WHO 1998). It can also be used on the evaluation of changes in the production process, vaccine stocks and methodology. Other use for this method would be the comparison among the $B$. pertussis strains used in the vaccine formulation and the bacterial isolates from the population, to verify the lot-to-lot consistency, prelicensing assays and to bring light to the better knowledge of the components involved in B. pertussis infection and immune response (Corbel et al. 1999a,b, Andre et al. 2000,Vandebriel et al. 2003). The humoral and cellular immunity plays an important role for complete elimination of the infection in mice in INM but not in ICM potency test (Atkinson et al. 2000, Coutte et al. 2003). Previous reports also demonstrated a high correlation between the 
lung clearance in mice and the efficacy of the vaccine in children (Mills et al. 1993, 1998, Watanabe et al. 2002a,b). Systemic and local reactions (infection of respiratory tract, pneumonia, and leucocytosis including the reversions of the disease) in INM test approaches to what happen in human pertussis disease.

During the last two decades of the XX century, the number of reported cases increased in all age groups, including adolescents and adults, indicating resurgence of the disease in developed countries. Since mass immunization in Brazil began only in the 1980s, one cannot rule out the endemicity and the possibility of pertussis reemergence in the near future. Therefore, it is important that public health services closely monitor the epidemiological situation of pertussis in order, if necessary, to rapidly update the current immunization strategy (Luz et al. 2003). Thus research for less expensive and simple potency tests for pertussis vaccines would be helpful. In the present study, we examined the INM using conventional NIH mice for its potential utility in assessment of the immunity induced by cellular and acellular vaccines combinations.

\section{MATERIALS AND METHODS}

Animals - Conventional NIH Mice, both sexes, 12 to $16 \mathrm{~g}$, from Cecal-Fiocruz (Centro de Criação de Animais de Laboratório) were used in these experiments. All the procedures using laboratory animals were taken following SOP: Boas Práticas em Experimentação Animal no. 65.3340.022, INCQS (2005). The study protocol, number P0133-02, was approved by the Fiocruz Ethic Commitee for Animal Experiments.

Bacterial reference strain and culture medium - B. pertussis 18323 (ATCC 9797 Type strain) from American Type Culture Collection was obtained from the Laboratory of References Microorganisms-INCQS. Bacterial strain was consecutively cultured on sheep blood BordetGengou (BG) medium added $400 \mathrm{mg} / \mathrm{l}$ cycloheximide (McGinnis 1980) for $48 \mathrm{~h}, 24 \mathrm{~h}$, and $18 \mathrm{~h}$ and then used in both ICM and INM experiments (WHO 1990).

Vaccines - The reference pertussis vaccine used on this study for both INM and ICM methods was Lot $10-$ freeze-dried non-adsorbed preparation - from Federal and Drugs Administration - FDA/US. The vaccines samples used in the INM included pertussis whole cell $(\mathrm{Pw})-$ adsorbed preparation - lot produced in Butantan Institute (strain: 137 - AGG I, II, III, IV, V and VI from NIH/US) - SP, Brazil and Acellular pertussis vaccines $(\mathrm{Pa})-\mathrm{Eu}-$ rope. $\mathrm{Pa}$ lots were divided into $\mathrm{Pa} 3$ and $\mathrm{Pa} 4$ due to preparation differences per dose ( $\mathrm{Pa} 3-25 \mu \mathrm{g}$ of Pertussis toxin, $25 \mu \mathrm{g}$ of FHA, and $8 \mu \mathrm{g}$ of Pertactin; Pa $4-10 \mu \mathrm{g}$ of Pertussis toxin, $5 \mu \mathrm{g}$ of FHA, $3 \mu \mathrm{g}$ of Pertactin, and $5 \mu \mathrm{g}$ of Fimbriae). Experiments included negative control groups of mice inoculated with saline solution $0.85 \%$. All vaccines tested were previously approved by the national authority - INCQS-Fiocruz and Brazilian National Immunization Program.

Evaluation of the immunity induced by cellular and acellular vaccines combinations ICM - The Kendrick test was performed as described by WHO (1990). In brief, groups of NIH mice from local sources were immunized intraperitoneally with serial dilutions of the test and reference vaccine preparation. The intracerebral challenge was carried out two weeks after immunization. The number of protected mice was recorded up to two weeks after challenge. The potency of the test vaccine was calculated by means of probitos assay against the reference preparation.

INM-NIH conventional mice - Experiments were carried out in triplicate and based in methods previously described by Guiso et al. (1999) and Guiso (2001) using conventional mice. Similarly to the metodology described for the Kendrick test, NIH mice groups, 12 mice each group were inoculated intraperitoneally with $0.5 \mathrm{ml}$ from the $1 / 4$ dilution of the test and reference vaccines (Mills et al. 1998, Xing et al. 1999, Andre et al. 2000, Canthaboo et al. 2000b, Watanabe et al. 2002a,b). A booster dose was given two weeks later. Two weeks after the booster dose, the animals were anesthetized by ether inhalation and $25 \mu 1$ of B. pertussis suspension $\left(10^{8} \mathrm{CFU} / \mathrm{ml}\right)$ was instilled into one nostril of each mouse. Infected mice were killed by cervical dislocation -3 animals per cage -1 day, 7 days and 15 days post-challenge. Groups of 12 mice inoculated with saline solution were also included as control in all performed experiments.

After ablation, the right lung of each animal was homogenized in $1 \mathrm{ml}$ sterile $0.85 \% \mathrm{NaCl}$ saline solution, 10 fold serial dilutions of homogenates were plated on BG and incubated at $36^{\circ} \mathrm{C}$ for five days for determination of viable counts (Colony Forming Units - CFU). The mean number and standard deviation of the log of CFU/mice was calculated for each time point.

Statistical analysis - Each lot of reference, Pw, Pa3, $\mathrm{Pa} 4$ vaccines was tested at least four times. Analysis of variance (Anova) and covariance (Ancova) were carried out using the Statistica for Windows - Release 4.0 Program - @Statsoft Inc. 1993 to compare means of experiments. Values of $p<0.05$ were considered statistically significant.

\section{RESULTS AND DISCUSSION}

In mice, the ICM test shows only local reactions, mainly a fatal cerebral edema, which is the base for the dose-response curve to access the potency of Pw vaccine (Canthaboo et al. 2000b). The current potency ICM test for $\mathrm{Pw}$ vaccines has considerable disadvantages as it uses a severe challenge procedure and the results tend to show significant intra and interlaboratory variation. The results from the evaluation of potency of different lots of $\mathrm{PW}$ vaccines by ICM are represented in Fig. 1. Interlaboratorial differences were observed among different lots of Pw analyzed mainly in lots G, L, M, Q, R, and V. Evaluation by the Kendrick test also showed significant intralaboratory variation. The minimum requirements $(\geq 8$ $\mathrm{UI} / \mathrm{ml}$ ) were reached in all lots tested. The statistical analysis showed similarities between data $(\mathrm{UI} / \mathrm{ml})$ obtained by the ICM-local test (mean:13.27 \pm 3.82 ) and ICM-producer test (mean:14.29 \pm 4.63 ) (Anova; $p$ 0.84).

In the INM the number of animals and the distress is much lower than ICM challenge (Xing et al. 1999). In at- 


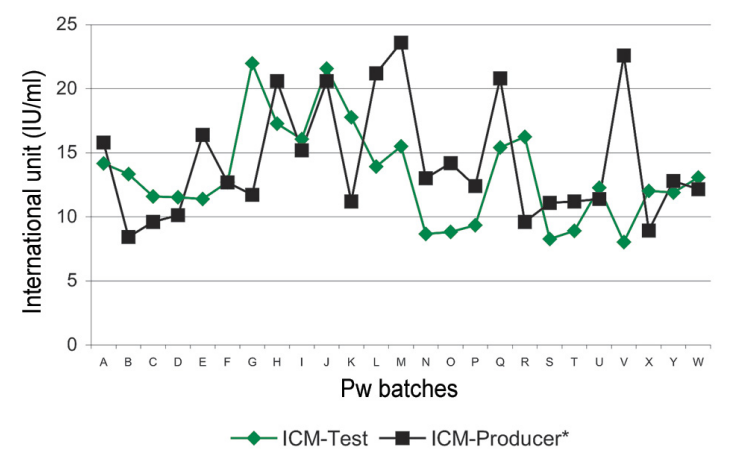

Fig. 1: graph of the results of intracerebral challenge (ICM) conventional mice of $\mathrm{Pw}$ vaccines. Each point represents the value of the potency of each tested lot $(\mathrm{UI} / \mathrm{ml})$ able to protect the mice. The values were defined in face of reference vaccine. Assays performed in groups (A to W) of 60 mice. * Results given by Butantan Institute, São Paulo.

tempt to turn this respiratory model less expensive and easily handled in non-aseptic environmental conditions, we evaluated the use of conventional NIH mice in INM challenges. The INM model may be used to compare the immunity induced by different vaccines but cannot quantify their potency for this moment.

Here in, we also would like to emphasize that INMconventional NIH mice test procedures required the following conditions: (i) selection of $B$. pertussis strain and growth conditions for enhancement of the expression of adhesins and toxins; (ii) use of fresh bacterial suspensions since frozen B. pertussis cells were unable to infect the respiratory tract of mice; (iii) use of cycloheximide for inhibition of contamination of culture medium.

Results of statistical analysis (Table I) indicate similarities in number of viable bacteria (CFU) obtained from

\section{TABLE I}

Number of viable (CFU) Bordetella pertussis cells obtained from mice control group 1,7 , and 15 days after infection

\begin{tabular}{lccc}
\hline & \multicolumn{3}{c}{ Log CFU } \\
\cline { 2 - 4 } Mice control groups $^{a}$ & 1st day & 7th day & 15th day \\
\hline C1 & 3.92 & 4.34 & 2.33 \\
C2 & 4.24 & 4.75 & 4.44 \\
C3 & 5.50 & 4.30 & 4.12 \\
C4 & 4.33 & 4.11 & 4.21 \\
C5 & 4.32 & 4.26 & 4.21 \\
C6 & 4.13 & 4.21 & 3.80 \\
C7 & 4.19 & 4.78 & 3.79 \\
C8 & 4.81 & 4.56 & 3.63 \\
C9 & 5.22 & 4.60 & 4.13 \\
C10 & 4.19 & 4.77 & 3.70 \\
C Mean & $4.48 \pm 0.51$ & $4.46 \pm 0.23$ & $3.8 \pm 0.59$ \\
\hline
\end{tabular}

CFU: Colony Forming Units; experiments were done in triplicate; $a$ : mice inoculated with saline solution; ANCOVA, $p$ 0.1919; ANOVA among the groups of days after challenge 1 and 7 and $15, p 0.006868$; between days 1 and 7, $p 0.9246$; between days 7 and $15, p 0.0060$. lungs of infected mice within unimmunized control groups (Ancova; $p$ 0.1919) obtained by INM.

The number of viable bacteria obtained from lungs of infected mice immunized with reference vaccine LOT 10/ FDA in INM is represented in Table II. Statistical differences (Anova; $p$ 0.0000) were found among CFUs obtained from mice lungs at 1,7 , and 15 day(s) post infection: (4.03 $\mathrm{Log} \mathrm{CFU}>2.95 \mathrm{Log} \mathrm{CFU}>0.12 \mathrm{Log} \mathrm{CFU}$, respectively). Comparative analysis among mice groups (R1 to R8) revealed similar results (Ancova; $p$ 0.05) demonstrating the reproducibility of the INM-conventional NIH mice. As shown in Tables I and II, the mean number of viable bacteria in the mice control group (4.48Log CFU) was similar to mice group inoculated with reference vaccine (4.03Log CFU) at the first day after INM-conventional NIH mice challenge. In contrast to the control mice group (4.46Log CFU), a lower number of viable bacteria in the lungs of vaccinated mice were observed in the 7th day after challenge (2.95Log CFU). Increased differences were detected at the 15 th day after challenge with 0.12Log CFU and 3.83Log CFU in vaccinated and control group, respectively. These results showed the ability of the INMconventional NIH mice to verify the efficacy of the reference pertussis vaccine to promote lung clearance.

The mean number of viable bacteria in Pw 1 to $\mathrm{Pw} 9$ mice groups inoculated with cellular pertussis vaccine is presented in Table III. Statistical analysis revealed similarities among results of assays performed with $\mathrm{Pw}$, indicating the reproducibility of the assays (Ancova, $p 0.2342$ ). The number of viable bacteria determined at the first day after infection with $B$. pertussis of $\mathrm{Pw}$ mice groups was 4.30 Log CFU. These results were similar to those obtained with the control mice group (Table I; 4.48Log CFU) and mice group immunized with the reference vaccine (Table II; 4.03 Log CFU). A decrease in number of viable bacteria was observed at the 7 th and 15 th days after infection, represented by $3.23 \mathrm{Log}$ CFU and 1.56Log CFU, respectively. Data reinforce the ability of the INM using conventional

\section{TABLE II}

Number of viable (CFU) Bordetella pertussis cells obtained from mice immunized with reference vaccine 1,7 , and 15 days after infection

\begin{tabular}{lccc}
\hline $\begin{array}{l}\text { Reference } \\
\text { vaccine mice } \\
\text { groups }\end{array}$ & \multicolumn{3}{c}{ Log CFU } \\
\cline { 2 - 4 } R1 & 1st day & 7th day & 15th day \\
\hline R2 & 4.67 & 2,80 & 0,00 \\
R3 & 3,18 & 3,00 & 0,00 \\
R4 & 3,84 & 3,48 & 0,00 \\
R5 & 4.70 & 3,49 & 0,00 \\
R6 & 4.23 & 2,34 & 0,00 \\
R7 & 4.77 & 3,64 & 0,00 \\
R8 & 3,60 & 2,47 & 1,00 \\
R Mean & $4,03 \pm 0.53$ & $2,95 \pm 0.46$ & $0.12 \pm 0.35$ \\
\hline
\end{tabular}

CFU: Colony Forming Units; experiments were done in triplicate; Reference vaccine (Lot 10/FDA) prepared at $10 \mathrm{IU} / \mathrm{ml}$; ANCOVA, $p$ 0.5424; ANOVA days 1 and 7 and 15, $p 0.00000$. 
NIH mice to verify the efficacy of Pw vaccine to promote lung clearance.

Table III reveals the number of viable bacteria $4.30 \mathrm{Log}$ CFU) determined at the first day after infection with $B$. pertussis of $\mathrm{Pw}$ mice groups. These results were similar to those obtained with reference vaccine mice groups (4.03 $\log$ CFU). A decrease in number of viable bacteria was observed at the 7 th and 15 th days after infection, represented by 3.23Log CFU and 1.56Log CFU for Pw group and 2.95Log CFU and $0.12 \mathrm{Log} C F U$ for reference group, respectively (Anova test p 0.0999 Table VI).

Table IV shows the mean number of viable bacteria in infected mice groups immunized with $\mathrm{Pa} 3$ vaccine. A decrease in the mean number of viable bacteria determined at the $1 \mathrm{st}, 7$ th, and 15 th days after infection with $B$. pertussis of $\mathrm{Pa} 3$ mice groups was 4.27Log CFU, 3.71 Log CFU, and $1.67 \mathrm{Log} \mathrm{CFU}$, respectively.

Table V present data of INM-conventional NIH mice immunized with component $\mathrm{Pa} 4$ vaccine. The mean number of viable bacterial cells determined at the $1 \mathrm{st}, 7 \mathrm{th}$, and 15 th days after infection was 4.34Log CFU, 4.28Log CFU, and 3.42Log CFU, respectively. These results were similar to those obtained with the mice control group.

\section{TABLE III}

Number of viable (CFU) Bordetella pertussis cells obtained from mice immunized with cellular pertussis vaccine $(\mathrm{Pw}) 1,7$, and 15 days after infection

\begin{tabular}{lccc}
\hline & \multicolumn{3}{c}{$\log$ CFU } \\
\cline { 2 - 4 } Pw mice groups & 1st day & 7th day & 15th day \\
\hline Pw 1 & 3.62 & 1.67 & 1.80 \\
Pw 2 & 4.79 & 3.78 & 2.77 \\
Pw 3 & 4.20 & 3.84 & 0.00 \\
Pw 4 & 4.76 & 3.88 & 0.00 \\
Pw 5 & 4.20 & 3.84 & 3.77 \\
Pw 6 & 4.30 & 2.91 & 2.47 \\
Pw 7 & 3.95 & 2.85 & 0.00 \\
Pw 8 & 4.25 & 2.91 & 1.00 \\
Pw 9 & 4.65 & 3.40 & 2.30 \\
Pw Mean & $4.30 \pm 0.38$ & $3.23 \pm 0.73$ & $1.56 \pm 1.38$ \\
\hline
\end{tabular}

CFU: Colony Forming Units; experiments were done in triplicate; ANCOVA, $p$ 0.2342; ANOVA, days 1 and 7 and 15, p 0.0000087.

\section{TABLE IV}

Number of viable (CFU) Bordetella pertussis cells obtained from lungs of mice immunized with three component acellular pertussis vaccine $\left(\mathrm{P}_{\mathrm{A}} 3\right) 1,7$, and 15 days after infection

\begin{tabular}{lccc}
\hline & \multicolumn{3}{c}{$\log$ CFU } \\
\cline { 2 - 4 } $\mathrm{P}_{\mathrm{A}}$ 3 mice groups & 1st day & 7th day & 15th day \\
\hline $\mathrm{P}_{\mathrm{A}}$ 3-1 & 3.49 & 3.66 & 0.00 \\
$\mathrm{P}_{\mathrm{A}} 3-2$ & 4.09 & 3.61 & 3.05 \\
$\mathrm{P}_{\mathrm{A}} 3-3$ & 5.14 & 3.38 & 2.00 \\
$\mathrm{P}_{\mathrm{A}} 3-4$ & 4.16 & 4.72 & 2.15 \\
$\mathrm{P}_{\mathrm{A}} 3-5$ & 4.48 & 3.19 & 1.15 \\
$\mathrm{P}_{\mathrm{A}}$ 3 Mean & $4.27 \pm 0.59$ & $3.71 \pm 0.21$ & $1.67 \pm 1.15$ \\
\hline
\end{tabular}

CFU: Colony Forming Units; experiments were done in triplicate; ANCOVA, $p 0.2779$.
Mean number of viable (CFU) B. pertussis cells obtained from lungs of NIH conventional mice inoculated with saline, reference vaccine, $\mathrm{Pw}, \mathrm{Pa} 3$, and $\mathrm{Pa} 4$ is represented in Fig. 2. Similar numbers of viable bacterial cells were observed at 1 st day after infection for all products tested. Significant differences observed at 7 th and 15 th day after infection yielded differentiation among products tested (Table VI).

Statistical analysis by Ancova test indicated the consistency $(p>0.05)$ of INM-conventional NIH mice for each product (saline control, p 0.1919 - reference vaccine, $\mathrm{p}$ $0.5424-\mathrm{Pw}, \mathrm{p} 0.2342$ and $\mathrm{Pa} 3, \mathrm{p} 0.2779$ ).

Significant differences were observed among INMconventional NIH mice results for $\mathrm{Pa} 4$ when compared with other products (Anova; reference vaccine, $p 0 ; \mathrm{Pw}, p$ 0.02 ; $\mathrm{Pa} 3, p$ 0.02) (Table VI). The performance of Pa4 vaccine at INM method brings it near to saline control group (Anova; p 0.26) (Fig. 2). Although significant, the difference between the data obtained with $\mathrm{Pa} 3$ and $\mathrm{Pa} 4$ component vaccines could be partially explained by differences in antigen concentration and the fact that the antigens are not similarly adsorbed on the adjuvant. For this reason it will be of importance to repeat experiments with INM to compare both vaccines by immunizing both vaccines with non diluted vaccines.

Comparative analysis revealed equivalent performance of INM-conventional NIH mice and the "gold standard" ICM method for determining the ability of cellular vaccine to protect mice against $B$. pertussis infection (unshown data).

\section{TABLE V}

Number of viable (CFU) of Bordetella pertussis cells obtained from lungs of mice immunized with four component acellular pertussis vaccine $\left(\mathrm{P}_{\mathrm{A}} 4\right) 1,7$ and 15 days after infection

\begin{tabular}{lccc}
\hline & \multicolumn{3}{c}{ Log CFU } \\
\cline { 2 - 4 } $\mathrm{P}_{\mathrm{A}}$ 4 mice groups & 1 st day & 7th day & 15 th day \\
\hline $\mathrm{P}_{\mathrm{A}} 4-1$ & 4,14 & 4,49 & 3.00 \\
$\mathrm{P}_{\mathrm{A}} 4-2$ & 3,62 & 3.69 & 2,82 \\
$\mathrm{P}_{\mathrm{A}} 4-3$ & 5.08 & 4,79 & 4,08 \\
$\mathrm{P}_{\mathrm{A}}^{4-4}$ & 4.54 & 4.18 & 3,78 \\
$\mathrm{P}_{\mathrm{A}} 4$ Mean & $4.34 \pm 0.61$ & $4.28 \pm 0.48$ & $3.42 \pm 0,60$ \\
\hline
\end{tabular}

CFU: Colony Forming Units; experiments were done in triplicate; ANCOVA, $p 0.00042$

\section{TABLE VI}

Statistical analysis by of the Intranasal model results among mice groups inoculated with saline, reference vaccine, cellular vaccine $(\mathrm{Pw})$, three component acellular vaccine $\left(\mathrm{P}_{\mathrm{A}} 3\right)$ and four component acellular vaccines $\left(\mathrm{P}_{\mathrm{A}} 4\right)$

\begin{tabular}{lcccc}
\hline & \multicolumn{4}{c}{$P$ values } \\
\cline { 2 - 5 } Products & $\mathrm{Pw}_{\mathrm{A}}$ & $\mathrm{P}_{\mathrm{A}} 3$ & $\mathrm{P}_{\mathrm{A}} 4$ & Saline \\
\hline Saline & 0.0001 & 0.0002 & 0.2600 & - \\
Reference vaccine & 0.0999 & 0.0021 & 0.0000 & 0.0000 \\
$\mathrm{PW}_{\mathrm{W}}$ & - & 0.7916 & 0.0286 & 0.0001 \\
$\mathrm{P}_{\mathrm{A}} 3$ & - & - & 0.0294 & 0.0002 \\
\hline
\end{tabular}

Experiments were done in triplicate; ANOVA ( $p 0.05)$ 


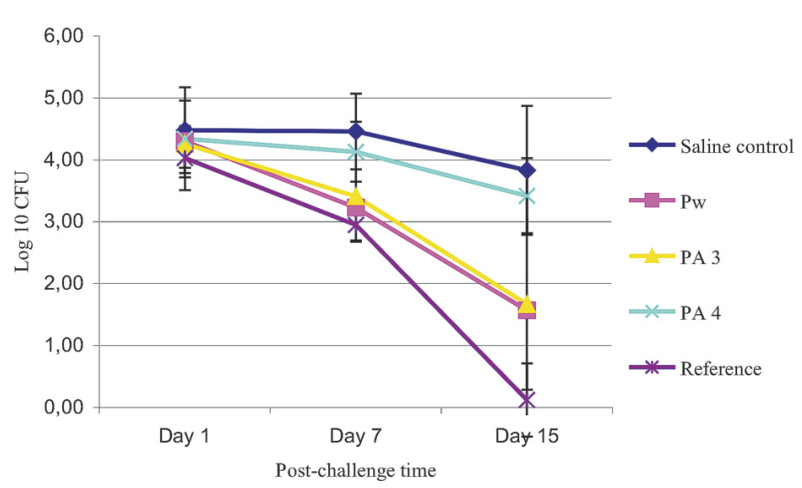

Fig. 2: graph of the mean of viable bacteria (Log CFU) in mice groups inoculated with saline solution, reference vaccine, cellular vaccine $(\mathrm{Pw})$, three component acellular vaccine $\left(\mathrm{P}_{\mathrm{A}} 3\right)$ and four component acellular vaccine $\left(\mathrm{P}_{\mathrm{A}} 4\right)$ at the intranasal challenge (INM) conventional mice.

The potency test using conventional NIH mice was also able to demonstrate a higher performance of cellular vaccines when compared with $\mathrm{Pa} 4$ acellular composition. The INM-conventional NIH mice differentiated Pa3 and $\mathrm{Pa} 4$ acellular pertussis compositions. Pa3 showed higher performance in face of $\mathrm{Pa} 4$. Data indicate the ability of INM-conventional NIH mice to demonstrate differences in antigen concentrations of pertussis compositions.

Thus the results indicate the INM - conventional NIH mice as an alternative method for evaluation of immunity induced by both cellular and acellular pertussis vaccines. Notwithstanding the results obtained on this and other studies, there is still a need of reference vaccines (wholecell and acellular, according to its composition) established by this method and with proved efficiency on field trials (Mills et al. 1998, Andre et al. 2000). Some conclusions concerning the differences between pertussis vaccines cannot be reached without repeating some experiments in a different way and comparing with the epidemiology in the field.

\section{REFERENCES}

Andre M, Poirier B, Bornstein N, Marmonier D, El Zaouk A, Fuchs F 2000. Key points for development of mouse imunogenicity test as potency assay for acellular pertussis vaccines. Biologicals 28: 217-225.

Atkinson W, Wolf C, Humiston S, Nelson R 2000. Epidemiology and Prevention of Vaccine-preventable Diseases, 6th ed., Department of Health e Human Services, CDC, Atlanta, p. 67-83.

Boas Práticas em Experimentação Animal 2005. In Manual da Qualidade: INCQS-Fiocruz, Rio de Janeiro (65.3440.002).

Canthaboo C, William L, Xing DKL, Corbel M 2000a. Investigation of cellular and humoral responses to whole cell and acellular pertussis vaccine. Vaccine 19: 637-643.

Canthaboo C, Xing DKL, Douglas A, Corbel M 2000b. Investigation of an aerosol challenge model as alternative to the intracerebral mouse protection test for potency assay of whole cell pertussis vaccine. Biologicals 28: 241-246.
Cherry JD, Grimprel E, Guiso N, Heininger U, Mertsola J 2005. Defining pertussis epidemiology clinical, microbiological and serologic perspectives. Pediatr Infect Dis $J 24$ : 25-34.

Corbel MJ, Xing DKL, Bolgiano B, Hockley DJ 1999a. Approaches to the control of acellular pertussis vaccines. Biologicals 27: 133-141.

Corbel MJ, Xing DKL, Kreeftenberg JG 1999b. Informal consultation with manufacturers and WHO Ad Hoc Working Group on Mouse protection models for acellular pertussis vaccines. Biologicals 27: 189-193.

Coutte L, Alonso S, Reveneau N 2003. Role of adhesin release for mucosal colonization by a bacterial pathogen. $J$ Exp Med 197: 735-742.

Eude CB, Guiso N, Thiberge S, Carletti G 1999. Intranasal murine model of Bordetella pertussis infection: II. Sequence variation and protection induced by three component acellular vaccine. Vaccine 7: 2651-2660.

Forsyth K, Tan T, Konig CHWV, Cairo JJ, Plotkin S 2005. Potential strategies to reduce the burden of pertussis. Pediatr Infect Dis J 24: 69-74.

Greenberg DP, Caro JJ 2005. Summary health and economic burden of pertussis. Pediatr Infect Dis J 24: 55-57.

Guiso N 2001. Whooping cough: agents and natural immunity. Med Mal Infect 31: 29S-38S.

Guiso N, Capiau C, Carletti G, Poolman J, Hauser P 1999. Intranasal murine model of Bordetella pertussis infection: I. Prediction of protection in human infants by acellular vaccines. Vaccine 17: 2366-2376.

Luz PM, Codeço CT, Werneck G 2003. The resurgence of pertussis in developed countries: a problem for Brazil as well? Cad Saúde Púb 19: 1209-1213.

McGinnis MR 1980. Laboratory Handbook of Medical Micology, Academic Press, New York, p. 523-585.

Mills KHG, Barnard A, Watkins J, Redhead K 1993. Cell mediate immunity to Bordetella pertussis: role of Th1 cells in bacterial clearance in a murine respiratory infection model. Infect Immun 61: 399-410.

Mills KHG, Ryan M, Ryan E, Mahon BP 1998. A murine model in which protection correlates with pertussis vaccine efficacy in children reveals complementary roles for humoral and cell-mediated immunity in protection against Bordetella pertussis. Infect Immun 66: 594-610.

Tan T, Plotkin S 2005. Controlling pertussis: consideration for the future. Pediatr Infect Dis J 24: 98.

Vandebriel RJ, Hellwig SMM, Vermeulen JP, Hoekman JHG, Dormans JAMA, Roholl PJM, Mooi FR 2003. Association of Bordetella pertussis with host immune cells in the mouse lung. Microbiol Pathog 35: 19-29.

Watanabe M, Komatsu E, Abe K, Iyana S, Sato T, Nagai M 2002a. Efficacy of pertussis components in an acellular vaccine, as assessed in a murine model of respiratory infection and a murine intracerebral challenge model. Vaccine 20: 429-1434.

Watanabe M, Komatsu E, Sato T, Masaaki N 2002b. Evaluation of efficacy in terms of antibody levels and cell-medi- 
ated immunity of acellular pertussis vaccine in a murine model of respiratory infection. FEMS Immunol Med Microbiol 33: 219-225.

WHO-World Health Organization 1990. Requirements for diphtheria, tetanus, pertussis and combined vaccines. International Requirements for Biological Substances. T R S 800: 87-149.
WHO-World Health Organization 1998. Guidelines for the production and control of the acellular pertussis component of monovalent or combined vaccines. T R S 878: 58-76.

Xing DKL, Das RG, Williams L, Canthaboo C, Tremmil J, Corbel MJ 1999. An aerosol challenge model of Bordetella pertussis infection as a potential bioassay for acellular pertussis vaccines. Vaccine 17: 565-557. 